\title{
Value-based Counseling
}

\section{Kultur und Religion als sinnstiftendes Element einer psychodynamischen Kurzzeitintervention}

\author{
Inge Missmahl \& Birte Brugmann
}

Psychotherapie-Wissenschaft 9 (1) 39-49 2019
www.psychotherapie-wissenschaft.info
CC BY-NC-ND
https://doi.org/10.30820/1664-9583-2019-1-39

Zusammenfassung: Value-based Counseling (VBC) ist aus der Praxiserfahrung der jungianischen Psychoanalytikerin Inge Missmahl entstanden, die sie vor allem in Afghanistan, aber auch in Sri Lanka, China, Haiti und der Ukraine machte. Die psychodynamische Kurzzeitintervention hat einen salutogenetischen Ansatz, der darauf abzielt, das Kohärenzgefühl und die Selbstwirksamkeit von Menschen wiederherzustellen. Dies geschieht im Rahmen eines nicht-direktiven, aber sorgfältig strukturierten Gesprächs. VBC beruht auf einem Menschenbild, das von einem inhärenten Bedürfnis des Menschen nach einem sinnerfüllten Leben ausgeht. Das Potenzial des Menschen zur Selbstentfaltung und sein Streben, dieses Potenzial zu nutzen, bildet die Grundlage für die Förderung menschlicher Selbstheilung. Der Beratungsansatz vermeidet eine Pathologisierung klinischer Symptome, denen intrapsychische oder interpersonelle Konflikte, traumatische Erlebnisse, ein disruptives soziales Umfeld oder schwierige Lebensübergänge wie Migration oder der Verlust von Lebensgrundlagen zugrunde liegen, und bemüht sich stattdessen um ein Verstehen der Bedeutung dieser Symptome als Ausdruck unbewältigten sozialen Stresses. VBC geht davon aus, dass sich einem Menschen, der sich seiner Situation, der damit verbundenen eigenen und fremden Wertehierarchie sowie der damit ausgelösten Gefühle bewusst wird, Handlungsspielräume für Veränderungen eröffnen. Als Ausgangspunkt für die Wahrnehmung der Welt und der damit verbundenen Gefühle wird Kultur und in diesem Zusammenhang auch Religion als eine sinnstiftende Ressource betrachtet, die gezielt im Counseling eingesetzt werden kann.

Schlüsselwörter: Afghanistan, Ausbildung, psychosoziale Beratung, Counseling, Deutschland, Entwicklungszusammenarbeit, Geflüchtete, Gesundheitssystem, Humanitäre Hilfe, Kollektivismus, Kultur, Krisengebiete, Migration, psychodynamische Kurzzeitintervention, Kurzzeittherapie, Menschenbild, niederschwellige Versorgung, Religion, Salutogenese, Selbstwirksamkeit, Sinn, Trauma, Wertvorstellungen

Die grosse Zahl an Menschen, die in Deutschland in den Jahren 2015 und 2016 Schutz suchten - vornehmlich aber nicht ausschliesslich vor dem Krieg in Syrien (Destatis, 2018) - hat das deutsche Gesundheitswesen vor neue Herausforderungen gestellt und unter anderem das Bewusstsein dafür gestärkt, dass Kultur ein wichtiger Faktor im Umgang mit Patient*innen ist. Das bezieht sich nicht nur auf Sprachbarrieren, sondern auch auf den kulturell geprägten Ausdruck psychischen Stresses sowie kulturell und unter Umständen religiös beeinflusste Wahrnehmungen körperlicher Beschwerden und Vorstellungen von geistiger Gesundheit. Eine Fülle von Literatur, die in letzter Zeit auf den Markt gekommen ist und sich mit kultursensiblen Ansätzen in der Psychotherapie beschäftigt, zeugt von diesem Trend (z. B. Preitler, 2016; v. Lersner \& Kizilhan, 2017; Koch, 2017; Liedl et al., 2017). Da die meisten Schutzsuchenden aus islamisch geprägten Ländern stammen, beschäftigen sich diese Ratgeber vornehmlich mit Herausforderungen, die durch die Begegnung christlich-jüdischer Traditionen mit dem Islam entstehen.

Der Bedarf nach kultursensibler Psychotherapie enstand jedoch nicht erst in den letzten Jahren. Bereits im Jahr 2011 lebten ungefähr 3,3 Millionen Muslim*innen in Deutschland, von denen über zwei Drittel türkische
Wurzeln hatten (Stichs, 2016). Sie wurden keineswegs ignoriert, wie beispielsweise ein Ratgeber aus dem selben Jahr zeigt (Rezapour \& Zapp, 2011), jedoch beschäftigten sie das deutsche Gesundheitssystem nicht in dem gleichen Mass wie die Ankunft von über einer Million Schutzsuchender seit 2015, die vorwiegend aus Syrien, dem Iran, Irak und Afghanistan stammen (BAMF, 2018). Nicht nur die grosse Zahl der Neuankömmlinge, sondern auch die besonderen gesundheitlichen und psychischen Probleme von Menschen, die Krieg und Vertreibung ausgesetzt waren, stellen das deutsche Gesundheitssystem und eine Vielzahl von Organisationen, die psychotherapeutsche oder psychosziale Dienste für Geflüchtete anbieten, vor besondere Herausforderungen.

Schutzsuchende in Deutschland stammen vornehmlich aus Ländern, deren Gesundheitssysteme bereits vor Ausbruch eines gewaltsamen Konfliktes oder vor einer Naturkatastrophe nur unzureichende Angebote im Bereich der psychischen Gesundheit machen konnten (Hijazi \& Weissbecker, 2014), und für die es eine enorme Herausforderung ist oder sein wird, diesen Zustand zu verbessern. Im Folgenden wird Value-Based Counseling (VBC) vorgestellt, eine Kurzzeitintervention für Menschen, die ihre Alltagstauglichkeit verloren haben, häufig Symptome von Depression, Angst und posttraumati- 
schen Belastungstörungen zeigen, und denen durch den psychodynamischen Beratungsansatz des VBC geholfen werden kann. Als ein niederschwelliges Angebot eignet VBC sich nicht nur für Krisengebiete, sondern auch als Teil eines mehrstufigen Versorgungssystems, der einer längeren Psychotherapie, die auf einer Diagnostik beruht, vorgeschaltet sein kann.

\section{Value-based Counseling}

VBC ist ein psychodynamischer kurztherapeutischer Beratungsansatz, der aus der Praxiserfahrung der jungianischen Psychoanalytikerin Inge Missmahl entstanden ist, die sie in Afghanistan und anderen Krisengebieten machte (Missmahl, 2006a, 2006b, 2010, 2016, 2018, i.D.; Ayoughi et al., 2012; Bohus \& Missmahl, 2017; Missmahl \& Ayoughi, 2017). VBC ist von C. G. Jungs Menschenbild und Verständnis der Psychodynamik geprägt und bezieht sich darüber hinaus auf Erkenntnisse von Victor Frankl, Carl Rogers und Albert Bandura, Aaron Antonovsky, Paul Watzlawick sowie Klaus Grawe. Es handelt sich dabei jedoch nicht um einen gezielten Eklektizismus, sondern um die Erfahrung, dass sich bestimmte Konzepte in der Praxis besonders bewährt haben.

VBC hat einen salutogenetischen Ansatz, der darauf abzielt, das Kohärenzgefühl (Antonovsky, 1997) und die Selbstwirksamkeit (Bandura, 2007) von Menschen wiederherzustellen. Dies geschieht im Rahmen eines nicht-direktiven (vgl. Rogers, 1994), aber sorgfältig strukturierten Gespräches. Antonovsky sah es als eine, wenn auch schwer umzusetzende Möglichkeit in der Therapie an, das Kohärenzgefühl eines Menschen zu stärken, indem Erfahrungen neu interpretiert werden (Antonovsky, 1997, S. 119). Grawes Studien zur Neuropsychologie (2004) legen jedoch nahe, dass Antonovsky mit seinem soziologischen Konzept weniger Persönlichkeitsmerkmal erfasste (Barwinski, 2016, S. 61ff.), als ein menschliches Bedürfnis nach Konsistenz, das für die Psychotherapie höchst relevant ist.

VBC beruht auf einem Menschenbild, das von einem inhärenten Bedürfnis des Menschen nach einem sinnerfüllten Leben ausgeht (vgl. Frankl, 2017 [2007]). Das Potenzial des Menschen zur Selbstentfaltung und sein Streben, dieses Potenzial zu nutzen, bildet die Grundlage für die Förderung menschlicher Selbstheilung. Der Beratungsansatz vermeidet eine Pathologisierung klinischer Symptome, denen intrapsychische oder interpersonelle Konflikte, traumatische Erlebnisse, ein disruptives soziales Umfeld oder schwierige Lebensübergänge wie Migration oder der Verlust von Lebensgrundlagen zugrunde liegen, und bemüht sich stattdessen um ein Verstehen der Bedeutung dieser Symptome als Ausdruck unbewältigten sozialen Stresses. VBC geht davon aus, dass sich einem Menschen, der sich seiner Situation, der damit verbundenen eigenen und fremden Wertehierarchie sowie der damit ausgelösten Gefühle bewusst wird, Handlungsspielräume für Veränderungen eröffnen.

\section{Anonymisierte Fallvignette Afghanistan}

Eine Mutter von vier Kindern wird von ibrer Familie zu einer Counselorin ${ }^{1}$ geschickt, weil sie als suizidal gilt. Die Fran berichtet, dass mit ibrer Verheiratung im Alter von 14 Jahren ein Konflikt zwischen ihrer Familie und der ibres Mannes beigelegt worden sei. Ihr Bräutigam sei zu der Zeit Mitte Dreissig gewesen. Die Klientin habe mit 16 Jahren ihr erstes Kind bekommen. Sie müsse die Hauptarbeit im Haushalt leisten und dürfe ihre eigene Familie nur selten sehen. Als sie mit den Jahren versucht habe, sich gegen diese Behandlung zu wehren, habe häusliche Gewalt gegen sie zugenommen. Sie befinde sich in einer verzweifelten Lage, weil sie das Haus ibrer Schwiegerfamilie verlassen wolle, aber in diesem Falle ihre vier Kinder zurücklassen müsse. Ihre Eltern seien aus gesellschaftlichen Zwängen heraus nicht in der Lage, sie wieder aufzunehmen, und der Aufenthalt in einem Frauenhaus sei ebenfalls keine Lösung, weil er sie in ihrem sozialen Umfeld zu einer Ausgestossenen machen würde. Die Klientin wirkt erschöpft und aggressiv.

Für die Klientin ist zunächst wichtig, dass sie mit einer Counselorin spricht, die nicht Teil ihres unmittelbaren sozialen Umfeldes ist, jedoch aus der gleichen Kultur stammt. Dadurch, dass sie von der Counselorin in ihrer Situation mit all ihren Gefühlen gesehen wird, kann sie sich wieder selbst spüren, was eine Voraussetzung dafür bildet, dass sie sich aus ihrer viktimiserten Position befreien kann. Gemeinsam mit der Counselorin reflektiert sie, welche Werte das Handeln ihrer Familie bestimmten, als sie sie verheiratete, was diese Zwangsehe für ihren Mann bedeutet, und wie diese konfliktreiche Situation das Leben ihrer Kinder beeinflusst. Auch ihre eigenen Werte werden im Gespräch reflektiert, wodurch der Klientin bewusst wird, dass sie ihre Kinder nicht verlassen will. Sie stellt fest, dass ihr Selbstverständnis vor allem darin besteht, ihren Kindern eine gute Mutter zu sein; und, dass sie ihrer leidvollen Erfahrung einen Sinn geben kann, indem sie Handlungsspielräume findet, in denen sie den Kreislauf der Gewalt in der Familie durchbrechen kann, um ihren Kindern ein anderes Aufwachsen zu ermöglichen, als sie es selbst erfahren hat.

VBC vermeidet eine Reduzierung sozial verursachten Leides auf eine individuelle Pathologie und ermöglicht stattdessen ein Empowerment, das Klient*innen dazu befähigt, Einfluss auf die belastende soziale Situation zu nehmen. Für VBC als einem kulturübergreifenden Beratungsansatz ist in diesem Zusammenhang besonders wichtig, dass Counselor*innen keine Lösungen für Probleme vorgeben, sondern Klient*innen darin unterstützen, eigene Lösungen zu entwickeln. In ihrer Ausbildung lernen Counselor*innen die Perspektive zu wechseln, um vorurteilslos die innere Situation der Klient*innen aus deren Sicht zu verstehen. Sie lernen, sich mit Annahmen über den inneren Zustand der Klient"innen und daraus folgenden Deutungen zurückzuhalten, um stattdessen mit

1 In der Praxis des VBC wurde das englische Wort «Counselor» eingedeutscht: Plural: «Counselor», weibliche Form «Counselorin». 
authentischem Interesse und empathischem Verständnis gemeinsam im Gespräch ein Verständnis der inneren Situation der Klientin oder des Klienten zu entwickeln.

In diesem Rahmen kann auf Augenhöhe verhandelt werden, was Watzlawick (2008 [2001], S. 38f.) die «Wirklichkeit zweiter Ordnung» genannt hat, das heisst die Bedeutung der Wirklichkeit, die wir mit unseren Sinnen wahrnehmen, und der wir in der Regel einen Sinn oder Wert zuschreiben. VBC kommt mit diesem Ansatz der strategischen Kurzzeittherapie nahe, die Watzlawick und Nordone (2008 [2001], S. 13; s. a. Gräff-Rudolph \& Sulz, 2014) als eine Intervention beschrieben,

«deren Ziel die Beseitigung der Symptome und die Lösung des vom Patienten dargestellten Problems ist. Dieser Ansatz ist weder eine Verhaltenstherapie noch eine oberflächliche Symptomtherapie, sondern eine Umdeutung und Veränderung der Wirklichkeitswahrnehmung und der sich daraus ergebenden Verhaltensweisen des Patienten.»

Im VBC vermeiden Counselor*innen Deutungen der erlebten Wirklichkeit der Klient"innen, die letztere von ihrer Kultur entfremden könnten. Der Einsatz von Counselor*innen aus dem selben Kulturkreis ermöglicht einen «kulturimmanenten» Umgang mit der Wirklichkeit, der das Potenzial hat, das Kohärenzgefühl und die Selbstwirksamkeit von Klient*innen zu stärken und Lösungen zu formulieren, die im jeweiligen sozialen Umfeld umsetzbar sind. Dafür explorieren Klient*innen und Counselor* innen gemeinsam kollektive, familiäre und persönliche Werte, die intrapsychischen oder interpersonellen Konflikten zugrunde liegen. Das strukturierte Gespräch erfolgt in sieben Schritten.

- Schritt 1: Analyse der Symptome und/oder des sich präsentierenden Problems

> Schritt 2: Analyse der psychosozialen Stressoren, die ursächlich mit den Symptomen in einem Zusammenhang stehen

> Schritt 3: Analyse des Zusammenhanges zwischen Symptomen und Stressoren und des dominierenden Grundgefühls; Psychoedukation

Ziel dieser ersten drei Schritte ist es, die Bedeutung des Symptoms in einem gegebenen kulturellen Kontext zum gegenwärtigen Zeitpunkt, aber auf der Grundlage des biografischen Hintergrunds des Klienten oder der Klientin zu verstehen. Dies dient nicht der Absicht, das Symptom zum Indikator für eine Diagnose zu machen und den Zustand der Klientin oder des Klienten zu pathologisieren, sondern es zielt auf die Erarbeitung eines gemeinsamen Verständnisses ihrer oder seiner inneren Situation ab. VBC sieht das Symptom als einen Träger für eine potenzielle Entwicklungsenergie auf, die erkannt sein will, und die es zu fördern gilt. Dazu wird ein Narrativ der persönlichen Geschichte der Klientin oder des Klienten entwickelt, das bis zu dem Punkt in der Vergangenheit zurückgeht, der als Auslöser für die im Augenblick gezeigte Symptomatik erkannt wird. Wichtig ist hierbei das Verständnis des dominierenden Grundgefühls der Klientin oder des Klienten, das die Alltagstauglichkeit einschränkt.

$>$ Schritt 4: Entwicklung der Hauptbeschwerde: Welches dominierende Grundgefühl beeinträchtigt die Alltagsfunktionalität am meisten?

$>$ Schritt 5: Entwicklung eines Lösungsansatzes für die Hauptbeschwerde, der mit dem kulturellen sozialen Umfeld sowie den persönlichen Werten der Klientin oder des Klienten vereinbar ist

Die Schritte 4 und 5 beziehen sich auf das Hier und Jetzt im Alltag der Klient"innen.

\section{> Schritt 6: Triage, psychosoziale Interventionen entspre-} chend der Indikation, Umsetzung der Lösung

Schritt 6 beschreibt Interventionstechniken, die für eine Umsetzung der Lösung geeignet sind, beziehungsweise Klient*innen, die eine medizinisch/psychiatrische Behandlung brauchen, oder besteht in einer Überweisung. Kommt es nicht zu einer Überweisung, werden je nach Indikation unterschiedliche Interventionstechniken aus verschiedenen therapeutischen Schulen angewendet. Diese umfassen unter anderem verhaltenstherapeutische Interventionen, eine Trauma-Guideline, Konfliktlösungsansätze, Trauerarbeit und Massnahmen gegen geschlechtsbezogene Gewalt.

> Schritt 7: Verankerung, Zusammenfassung, Ausblick

Ziel des letzten Schrittes ist es, den Erkenntnisprozess, den der Klient oder die Klientin durchlaufen hat, in dessen oder deren Bewusstsein so zu verankern, dass dieser Mensch in zukünftigen schwierigen Lebenssituationen besser gerüstet ist, die Situation selbst zu meistern.

$\mathrm{Da}$ für diesen Beratungsansatz Menschen aus verschiedenen Kulturkreisen mit dem Ziel zu Counselor*innen ausgebildet werden, muttersprachlich für ihre Landsleute tätig werden zu können, ist er in seiner Anwendung nicht den Beschränkungen interkultureller Kommunikation unterworfen. Stattdessen betrachtet VBC Kultur und in diesem Zusammenhang auch Religion als Ausgangspunkt für die Wahrnehmung der Welt und der damit verbundenen Gefühle als Reaktion sowie als eine sinnstiftende Ressource, die gezielt im Counseling eingesetzt werden kann.

\section{VBC im Kontext psychodynamischer Kurzzeittherapien}

Psychotherapeutische Angebote in Krisengebieten müssen praxistauglich sein, ebenso wie in Entwicklungs- und Schwellenländern, deren Alltag von Schwierigkeiten aller Art geprägt ist. Dies gilt für die Ausbildung qualifizierter Therapeut*innen ebenso wie für die Möglichkeiten, gängige Therapieformen zu finanzieren. Angesichts der Tatsache, dass sogar in Deutschland als einem Industrie- 
land mit einer flächendeckenden Krankenversicherung der Bedarf die bereitgestellten Mittel übersteigt (BPtK, 2018), stellt sich die ernsthafte Frage, wie eine niederschwellige Versorgung im Bereich der psychischen Gesundheit unter weniger guten Voraussetzungen aussehen kann.

\section{Anonymisierte Fallvignette Afghanistan}

Ein Klient wendet sich an einen Counselor mit der Erklärung, er leide unter Kopfschmerzen und Konzentrationsmangel, Antriebslosigkeit und Sinnverlust. Er sei vor einigen Jahren bei einem Anschlag schwer verletzt worden, verdanke es jedoch seiner Position als Projektleiter einer internationalen Organisation, dass er zur Behandlung seiner Verletzungen nach Indien habe reisen können, wo ihm auch Gespräche mit einem Psychologen angeboten worden seien. Er habe mit Gottes Hilfe den Anschlag überlebt und gelte als geheilt. Umso schwieriger sei es für ihn zu akzeptieren, dass es ihm jetzt nicht gut gehe.

Im Gespräch stellt sich heraus, dass der Klient mit seinen Eltern, seiner Frau, den gemeinsamen Kindern und weiteren finanziell abhängigen Familienmitgliedern unter einem Dach lebt. Zu Eheproblemen kämen ungezogene Kinder, die der Klient massregeln müsse. Zwar tue es ihm im Nachbinein leid, wenn er sie geschlagen habe, und er leide darunter, wenn sie sich von ihm fernhielten, nur wisse er sich nicht anders zu helfen. Er habe das Gefühl, dass seine Familie ihn nicht mehr respektiere und seine Rolle als Familienoberhaupt infrage stelle. Der Lehrer seines Sobnes habe ihn angesprochen, weil der Sohn sich zunehmend häufig mit anderen Kindern streite und prügele. Auch seine Frau habe sich verändert und er wisse nicht, ob er ibr noch vertrauen könne. Auf der Arbeit sei die Situation ebenfalls schwierig. Er habe das Gefühl, dass ihn seine Mitarbeiter nicht mehr respektieren würden und wisse nicht mehr, wie er die Kontrolle über das Projekt behalten könne.

Der Klient bringt diese Situation nicht mit dem Anschlag in Verbindung. Im Gespräch mit dem Counselor wird ihm deutlich, dass die negative Entwicklung in seinem Familienleben ursächlich mit einem Gefühl der allgemeinen Verunsicherung und seiner dauernden Übererregtheit im Zusammenhang steht, und dass diese Verunsicherung und Übererregtheit eine Reaktion auf den Anschlag ist, den er überlebt hat. Dieser Zustand ist mit seinem Selbstverständnis als Familienoberhaupt, dessen Aufgabe es ist, die Familie zu ernähren und zu schützen, nicht vereinbar. Der Klient erkennt, dass er versucht hat, seinen inneren Kontrollverlust und seine generelle Verunsicherung über eine stärkere Kontrolle in der Familie zu kompensieren. Die Erleichterung über dieses neue Verständnis der Situation drückt sich im Moment der Erkenntnis unmittelbar in der Körpersprache des Klienten aus.

Es handelt sich bei der hier geschilderten Problematik keinesfalls um einen Einzelfall. In Afghanistan lässt sich immer wieder beobachten, dass sich die Interaktion in einer Familie verändert, wenn ein Familienoberhaupt nach einem traumatischen Erlebnis unter Symptomen von Hyperarousal leidet, emotionale Nähe vermeidet und kompensatorisch zum Kontrollverlust über seine Gefühle das Bedürfnis entwickelt, entsprechend seines Rollenverständnisses nach aussen hin mehr Kontrolle auszuüben. Gewalt wird in die Familie hineingetragen und von dort aus zurück in das soziale Umfeld, wie am Beispiel des Verhaltens des Sohnes in dem geschilderten Fall deutlich wird.

Eine Voraussetzung dafür, einen Fall wie diesen im Rahmen einer Kurzzeitintervention erfolgreich angehen zu können, war der Verzicht auf eine Diagnose und damit auf das Machtgefälle zwischen Counselor und Klient. Stattdessen bemühen sich Counselor und Klient in einer symmetrischen Beziehung um ein gemeinsames Verständnis der Bedeutung des Symptoms im persönlichen biografischen sowie sozialen und kulturellen Kontext des Klienten. Zudem wurden Beschränkungen, denen die Kommunikation zwischen Klient und Counselor in einer interkulturellen Beratung - so kultursensibel sie auch sein mag - unterworfen gewesen wäre, umgangen, indem ein muttersprachlicher Counselor desselben Geschlechts darin geschult worden war, ein vertrauensvolles Verhältnis mit Klienten aus demselben Kulturkreis aufzubauen.

Für Kurzzeittherapien müssen gewisse Voraussetzungen gegeben sein, die Hanna Levenson (2017, S. 6-13) folgendermassen zusammenfasst: Die Therapie muss sich sehr früh im Prozess auf ein bestimmtes Ziel konzentrieren, und es muss ein gutes Verhältnis zwischen Patient* in und Therapeut*in bestehen. Hierzu gehört Einigkeit darüber, dass das Verhältnis auf einen bestimmten Zeitraum begrenzt sein wird, sowie die Erwartungshaltung, dass die Kurzzeittherapie Veränderungen anstösst, die sich längerfristig auswirken werden. Ein weiteres Merkmal psychodynamischer Kurzzeittherapien besteht darin, dass das Verständnis der inneren Situation der Klientin oder des Klienten ein psychodynamisches ist, Interventionen jedoch Elemente aus anderen therapeutischen Schulen beinhalten können wie zum Beispiel die kognitive Verhaltenstherapie.

VBC erfüllt zwar die von Levenson beschriebenen Voraussetzungen, fällt jedoch bei ihr unter «Ultrakurzzeittherapien» oder "gesteuerte Versorgungs-Therapien» (managed care therapy; ebd., S. 57), weil VBC mit einer Regelzeit von drei bis fünf Sitzungen auf weniger als den von Levenson empfohlenen acht bis 25 Sitzungen angelegt ist. Levenson impliziert, dass es für Therapeut*innen nicht möglich ist, in so kurzer Zeit eine Beziehung mit Klient*innen aufzubauen, wodurch eine wesentliche Voraussetzung für eine wirksame Kurzzeittherapie fehle. Hier wird jedoch von einem letztendlich vertikalen Verhältnis zwischen Therapeut* in und Patient*in ausgegangen, in dem die Deutungshoheit über Symptome und Stressoren bei der Therapeutin oder dem Therapeut liegt. Dieses Verhältnis besteht bei VBC nicht.

Die Wirkung des VBC konnte im Rahmen einer Studie in einer Klinik in Nordafghanistan nachgewiesen werden. Der Beratungsansatz führte zu einem signifikanten Rückgang der Symptomatik und der psychosozialen Stressoren der Teilnehmer*innen (Ayoughi et al., 2012; Ayoughi, 2013). Eine vom Bundesministerium für Gesundheit finanzierte Wirksamkeitsstudie, welche die Wirksamkeit des Beratungsansatzes für Geflüchtete und Menschen mit Migrationshintergrund aus Afghanistan, Syrien und Afrika untersucht, ist gegenwärtig in Arbeit. 
Erfahrungen mit VBC in Afghanistan, Sri Lanka, China, Haiti und der Ukraine, aber auch in Deutschland legen nahe, dass Grawes Definition der Wirkfaktoren von Psychotherapien kulturübergreifend gelten (siehe Tabelle 1).
Als ein psychodynamischer kurztherapeutischer Beratungsansatz ist VBC provokant, weil er sich gegen gängige Auffassungen wendet (siehe Tabelle 2).
Wirkfaktoren von Therapien (nach Grawe, 2005, S. 7)

Motivationale Klärung: "sie fördern mit geeigneten Maßnahmen, dass der Patient ein klareres Bewusstsein der Determinanten seines problematischen Erlebens und Verhaltens gewinnt.»

Problemaktualisierung: "Sie machen die Probleme, die in der Therapie verändert werden sollen, dem Patienten unmittelbar erfahrbar.»

Problembewältigung: "Sie unterstützen den Patienten mit bewährten problemspezifischen Maßnahmen aktiv darin, positive Bewältigungserfahrungen im Umgang mit seinen Problemen zu machen."

Ressourcenaktivierung: "Sie nutzen Eigenarten, welche die Patienten in die Therapie mitbringen, als positive Ressourcen für das therapeutische Vorgehen. Sie nutzen also vorhandene motivationale Bereitschaften und Fähigkeiten der Patienten.»

Therapiebeziehung: «Bei allen Therapien trägt darüber hinaus die Qualität der Therapiebeziehung signifikant zu einem besseren oder schlechteren Therapieergebnis bei.»
Value-based Counseling

Schritt 3: Analyse des Zusammenhanges zwischen Symptomen und Stressoren und des dominierenden Grundgefühls; Psychoedukation

Schritt 4: Entwicklung der Hauptbeschwerde:

Welches dominierende Grundgefühl beeinträchtigt die Alltagsfunktionalität am meisten?

Schritt 5: Sobald Klient*innen ein Bewusstsein für ihre Situation entwickelt haben, eröffnen sich ihnen Handlungsspielräume. Sie können mit Unterstützung der Counselor*innen erkennen, welche Massnahmen zu einer für sie sinnvollen und ihrem kulturellen Umfeld angepassten Verbesserung der Situation führen können. Zu diesem Zweck werden eigene Ressourcen bewusst gemacht, entwickelt und aktiviert.

Counselor*innen begegnen ihren Klient*innen mit bedingungsloser Wertschätzung, authentischem Interesse, Empathie und dem Wunsch, helfen zu wollen, in einer symmetrischen Beziehung.

Tab. 1: Grawes Definition der Wirkfaktoren von Psychotherapien im Vergleich zu VBC

\begin{tabular}{|l|l|}
\hline \multicolumn{1}{|c|}{ Gängige Auffassungen } & \multicolumn{1}{c|}{ Value-based Counseling } \\
\hline $\begin{array}{l}\text { Ein mehrjähriges Psychologiestudium ist eine } \\
\text { unabdingbare Voraussetzung für eine erfolgreiche } \\
\text { Durchführung von psychodynamischen Interventionen. }\end{array}$ & $\begin{array}{l}\text { Die klare Strukturierung des Gespräches und die symmetrische } \\
\text { Beziehung zwischen Counselor*in und Klient*in ermöglicht die } \\
\text { Schulung von Menschen zu Counselor*innen, die zwar eine Vorbil- } \\
\text { dung besitzen, aber nicht notwendigerweise Psychologie auf inter- } \\
\text { nationalem Niveau studiert haben, innerhalb eines Jahres. Bedin- } \\
\text { gung ist ein Selbsterfahrungsanteil in der Weiterbildung von 50\%. }\end{array}$ \\
\hline $\begin{array}{l}\text { In einem interkulturellen Setting wiegt der Vorteil } \\
\text { einer psychotherapeutischen Ausbildung nach } \\
\text { internationalen Standards die Nachteile auf, die } \\
\text { auf der Beziehungsebene zwischen Therapeut*in } \\
\text { und Patient*in dadurch entstehen, dass die } \\
\text { Kommunikation auch bei einer kultursensiblen } \\
\text { Beratung eingeschränkt ist. }\end{array}$ & $\begin{array}{l}\text { Anstatt Therapeut*innen aus der «internationalen Gemein- } \\
\text { schaft» in der kultursensiblen Anwendung ihrer Techniken } \\
\text { für Auslandseinsätze zu schulen, werden muttersprachliche } \\
\text { Counselor*innen aus demselben Kulturkreis dazu befähigt, in } \\
\text { kurzer Zeit eine Beziehung zu Klient*innen aufzubauen und zu } \\
\text { unterhalten (intrakulturell statt interkulturell). Dies wird von } \\
\text { einem Matching unterstützt, das z. B. dafür sorgt, dass i.d. R. }\end{array}$ \\
\hline $\begin{array}{l}\text { Diagnosen sind notwendig, um } \\
\text { a) den Zugang zu Hilfsangeboten zu rechtfertigen } \\
\text { und zu regulieren, } \\
\text { b) Erfolge messen zu können. }\end{array}$ & $\begin{array}{l}\text { Auf Diagnosen wird verzichtet, um } \\
\text { a) eine Beziehung zwischen Counselor*innen und Klient*innen } \\
\text { auf Augenhöhe zu ermöglichen, } \\
\text { b) das Selbstwertgefühl von Klient*innen nicht durch } \\
\text { das Werturteil «krank» zusätzlich zu belasten, } \\
\text { c) Definitionen von Krankheiten zu vermeiden, die in anderen } \\
\text { Kulturen anders wahrgenommen werden, und } \\
\text { d) um sozialen Stress nicht in eine individuelle Pathologie } \\
\text { umzudeuten. }\end{array}$ \\
\hline
\end{tabular}

Tab. 2: VBC im Vergleich zu gängigen Auffassungen über Psychotherapie 
Es sind die Ver- und Vorstösse in Bezug auf gängige Praktiken, die VBC für Einsätze ausserhalb deren Geltungsbereichs tauglich macht, seien es Flüchtlingslager an der syrischen Grenze oder das Gesundheitssystem eines Landes, das Jahrzehnte brauchen wird, um internationale Standards zu erreichen. Auch innerhalb ihres Geltungsbereiches kann VBC sinnvoll eingesetzt werden, wie im Falle eines Gesundheitssystems, das nicht auf die Bedürfnisse grosser Zahlen von Schutzsuchenden ausgerichtet ist. Dies erfordert, auf der Grundlage des Machbaren unter schwierigen Bedingungen auf internationale Standards hinzuarbeiten und Kritik an diesem Vorgehen auszuhalten.

\section{Das zugrundeliegende Menschenbild}

VBC beruht auf dem Menschenbild Jungs, dessen Selbstkonzept und Ziel der Individuation (das nicht mit dem soziologischen Begriff der «Individualisierung» gleichzusetzen ist) kulturübergreifender ist als das humanistische Menschenbild (Heine, 2005, S. 300f.), das stärker individualistisch geprägt davon ausgeht, dass der Mensch sich von gesellschaftlichen und religiösen Zwängen befreien muss, um zur Selbstverwirklichung zu gelangen. Im Prozess des Counselings soll vielmehr die innere Bewusstheit geschaffen und es sollen die persönlichen Ressourcen mobilisiert werden, die es dem Individuum ermöglichen, sein ihm innewohnendes Entwicklungspotenzial im gegebenen kulturellen und sozialen Kontext seiner Lebensrealität zu entfalten.

Therapeuten sollten das humanistische Menschenbild einer Behandlung in einem interkulturellen Kontext nicht zugrunde legen, ohne sich zuvor die folgende Frage beantwortet zu haben: Wie relevant ist die Selbstverwirklichung als Grundbedürfnis für kulturell bedingte Selbstkonfigurationen, die nicht individualistisch geprägt sind? Es steht der Vorwurf im Raum, dass es sich bei der Psychotherapie um ein neokolonialistisches Instrument handelt, mit dem vermeintlich in ihrer persönlichen Entwicklung gehinderte Menschen aus kollektivistischen Gesellschaften zu Individualisten gemacht werden sollen (vgl. z.B. Zaumseil, 2006; Kirmayer, 2007).

In Publikationen zur kultursensiblen Psychotherapie in Deutschland spielt die auf Hofstede (2001) zurückgehende Unterscheidung zwischen den Werten individualistischer und kollektivistischer Gesellschaften und daraus abgeleitete Selbstkonstruktionen eine wichtige Rolle, da kulturelle Missverständnisse den Erfolg einer Therapie gefährden können (vgl. z. B. Rezapour \& Zapp, 2011; v. Lersner \& Kizilhan, 2017). Muslimisch geprägte Gesellschaften werden in diesem Kontext in der Regel mit kollektivistisch geprägten Gesellschaften gleichgesetzt. Das gleiche gilt für die Unterscheidung zwischen einer independenten und einer interpendenten Selbstkonstruktion als einer Weiterentwicklung von Hofstedes Konzept (Zaumseil, 2006, S. 6ff.). Der oben geschilderte Fall der zwangsverheirateten Klientin stellt in diesem Kontext ein passendes Beispiel dar. In einer individualistisch geprägten Gesellschaft wäre eine Trennung die offensichtlichste und für viele die einzig menschenwürdige Lösung. Ihren Lebenssinn sieht die Klientin jedoch in ihrer Rolle als Mutter und Ehefrau im Rahmen ihrer Grossfamilie. Eine Therapeutin, die ihre Pflicht darin sähe, auf eine individualistisch geprägte Selbstkonstruktion hinzuarbeiten, die es ihrer Patientin erlauben würde sich von der Familie zu lösen, um eine Selbstverwirklichung nach westlichem Vorbild anzustreben, würde auf eine Veränderung im Leben der Patientin hinarbeiten, die sie von ihrem kulturellen Kontext entfremden und ihr mit grösster Wahrscheinlichkeit die Lebensgrundlage entziehen würde.

Selbst in Deutschland ist bei der Beratung von Schutzsuchenden in dieser Hinsicht grosse Vorsicht angebracht. Kommt es zu häuslicher Gewalt, werden Familien in der Regel zum Schutz von Frau und Kindern auf der Grundlage des Gewaltschutzgesetzes (BMFSFJ \& BMJV, 2017) getrennt untergebracht und der Frau die Scheidung empfohlen. Häufig wird die häusliche Gewalt durch den Verlust der sozialen Rolle des Mannes als Ernährer und Beschützer der Familie ausgelöst. Wird dem Verursacher der Gewalt ein solcher Kompensationsmechanismus im Gespräch bewusst, kann er der Gewalt oftmals ein Ende setzen und sein Familienleben dadurch erhalten. Eine Scheidung wird in vielen Fällen dem Selbstverständnis der betroffenen Frau nicht gerecht und stellt sie vor neue Probleme.

Vermutlich hatte Maslow (1943, S. 383) als Vertreter der humanistischen Psychotherapie keine kollektivistisch geprägten Gesellschaften im Sinn, als er das Bedürfnis nach Selbstverwirklichung als «den Wunsch nach Selbstentfaltung $[. .$.$] um alles zu werden, was im Rahmen der$ eigenen Möglichkeiten liegt» (Übers. d.A.) definierte, jedoch lässt sich die Definition auch auf interpendente Selbstkonstruktionen anwenden. VBC geht davon aus, dass das Bestreben nach Selbstentfaltung abhängig von kulturell geprägten oder persönlich entwickelten Werten für unterschiedliche Selbstkonstruktionen beziehungsweise kulturell bedingte Konfigurationen des Selbst (Kirmayer, 2007, S. 244ff.) eingesetzt werden kann, die nicht auf Egozentrismus oder Soziozentrismus beschränkt sein müssen, sondern ökozentrische oder kosmozentrische Aspekte enthalten können. Das in der heutigen Psychotherapie vorherrschende humanistische Menschenbild (Quitmann, 1996, S. 279ff.) eignet sich für VBC nur insoweit, als es Raum für solche Konzepte bietet.

\section{Die Rolle von Wertvorstellungen}

Die Psychologie tut sich im Umgang mit Wertvorstellungen schwerer als die Philosophie und Soziologie (vgl. Oerter, 2007, S. 556), hat jedoch mit Values Clarification eine psychotherapeutische Technik hervorgebracht, die hier von VBC abgegrenzt werden soll, um Missverständnisse zu vermeiden. Values Clarification wurde in der zweiten Hälfe des 20. Jahrhunderts für Kinder und Jugendliche in den USA entwickelt, die in einer individualistischen und liberalen Gesellschaft nach Orientierung suchten (Kirschenbaum, 2013, S. 5ff.). Dadurch erhielt 
die Technik einen spielerischen Charakter und vermittelt den Eindruck, als seien Werte etwas frei Wählbares, mit dem das Leben beliebig optimiert werden kann. Die Erfahrung mit VBC im kulturübergreifenden Kontext lehrt jedoch, dass Werte selten beliebig sind. In der Regel sind sie kulturell vermittelt und bildungsabhängig, werden meist im Rahmen von Generationskonflikten sichtbar und müssen im Prozess des Counselings oft mühevoll mit der sozialen Umgebung neu verhandelt werden. VBC hat daher mehr Gemeinsamkeiten mit Beratungsmethoden, die der Rogers-Schüler Charles Curran (1968, S. 3) als Value Counseling and Psychotherapy in sein Gesamtwerk integrierte, dass jedoch vor allem auf Erziehungsmethoden ausgerichtet war.

\section{Anonymes Fallbeispiel Afghanistan}

Eine Schülerin in einer Grossstadt sucht heimlich eine Counselorin auf. Sie habe sich mit ibren Freundinnen nach der Schule einen Spass daraus gemacht, so lange die eigene Telefonnummer mit einer veränderten Zabl zu wählen, bis ein junger Mann geantwortet habe. Es habe ein reger Austausch von Textnachrichten begonnen, gefolgt von regelmässigen Telefongesprächen. Man habe sich schliesslich getroffen, und es sei zu Intimitäten gekommen. Sie sei verliebt gewesen und habe sich wie die Heldinnen in den täglich im afghanischen Fernsehen laufenden türkischen Serien und Bollywood-Filmen gefühlt. Sie habe eine sexuelle Beziehung mit dem jungen Mann aufgebaut, nachdem er ihr die Heirat versprochen habe. Er habe sein Versprechen jedoch nicht eingehalten und drohe ihr damit, Bilder von ibr im Internet zu veröffentlichen und sie blosszustellen, wenn sie den Kontakt nicht abbreche. Ibre Eltern hätten inzwischen bemerkt, dass sie nicht mehr schlafe, nicht mehr esse und sich nicht mehr konzentrieren könne, und dass ibre Leistungen in der Schule deutlich nachgelassen hätten. Sie wisse keinen Ausweg.

In diesem Fall kollidieren die Werte der Familie, die ein strenges Tabu bezüglich vorehelicher Beziehungen beinhalten, mit dem Wunsch nach einem freieren Umgang mit dem anderen Geschlecht. Ausgelöst wurde dieser Konflikt durch die freie Verfügbarkeit von Bildern und Filmen, die zu Rollenmodellen für Jugendliche werden. In der traditionell geprägten afghanischen Gesellschaft können die Reaktionen auf vorehelichen Geschlechtsverkehr jedoch von gesellschaftlicher Ächtung bis hin zum Ehrenmord an der betroffenen Jugendlichen reichen. Im Prozess des Counselings wurden der Klientin die jeweiligen Werte bewusst, auf denen die unterschiedlichen Positionen beruhen, und es gelang ihr auf dieser Grundlage, ihre eigenen Werte neu zu bestimmen.

Rolf Oerter (2007, S. 555, 558, 563) definiert Werte "als Pflichten, Handlungsvorschriften, aber auch als abstrakte Begriffe wie Ehre, Wahrhaftigkeit, Güte», die als internale Konzepte für einen Menschen handlungsleitend sind. Er sieht in der Gegenüberstellung von individuellen und kollektivistischen Kulturen die wichtigste Unterscheidung bezüglich kultureller Wertordnungen. Jung (1990, S. 90) spricht von «Werturteilen», die das Gewissen fällt, letzteres ist «ein komplexes Phänomen, das einerseits aus einem elementaren Willensakt oder aus einem bewusst nicht begründeten Antrieb zum Handeln, anderenteils aus einem Urteil des vernünftigen Gefühls besteht». Das Gewissen unterteilt Jung (ebd., S. 97) in zwei Komponenten, die miteinander in Konflikt geraten können: das persönliche Gewissen und den gesellschaftlichen Sittenkodex, der gleichgesetzt wird mit traditioneller/konventioneller Moral. Eine Unterscheidung ist nach Jung (ebd., S. 99) jedoch oftmals schwierig, zumal vor allem das persönliche Gewissen grösstenteils im Unterbewusstsein liegt und das Gewissen «an sich ein autonomer psychischer Faktor» ist. Wertekonflikte können zu schwerwiegenden Belastungen für einen Menschen werden, der unter den Symptomen eines solchen Konfliktes leidet, sich der Ursache jedoch nicht bewusst ist. Ein wertebasierter Beratungsansatz kann daher nur erfolgreich sein, wenn es sich um einen psychodynamischen Ansatz handelt, der das erste Ziel hat, einen solchen Konflikt bewusst zu machen.

Der Umgang mit unterschiedlichen Wertvorstellungen stellt interkulturelle Psychotherapie vor enorme Anforderungen. Von Lersner und Kizilhan (2017) haben überzeugend dargelegt, dass im Rahmen einer interkulturellen Beratung, so kultursensibel sie auch erfolgen mag, lediglich eine Annäherung zwischen Therapeut*innen und Patient*innen erfolgen kann. Da es für Therapeut*innen in der Regel unmöglich ist, die Wahrnehmung der Realität auf der Basis tief empfundener Werte eines Menschen aus einer anderen Kultur umfassend nachzuvollziehen, müssen sie den mangelnden Zugang so weit wie möglich durch Offenheit kompensieren. Der Einsatz von Dolmetscher"innen erschwert eine Annäherung zusätzlich, da Vertrauen nicht nur zwischen zwei, sondern drei Menschen aufgebaut werden muss und die Kommunikation durch die Übersetzung noch stärker gefiltert wird, als es in einer interkulturellen Situation ohnehin der Fall ist.

Da VBC den Anspruch hat, auch unter schwierigen Bedingungen niederschwellig und effizient einsetzbar zu sein, erfolgt es ausschliesslich intrakulturell, das heisst muttersprachlich und durch Counselor*innen aus demselben Kulturkreis. In diesem Zusammenhang ist wichtig, dass ein Matching von Klient*innen und Counselor*innen soziokulturelle Barrieren berücksichtigt, die eine niederschwellige Beratung des jeweils anderen Geschlechtes in Kulturen mit stark ausgeprägten geschlechtsspezifischen Rollenverteilungen und Tabus schwierig machen würde. Da dem jeweils anderen Geschlecht in solchen Kulturen grundlegende Erfahrungen fehlen und gesellschaftliche Tabus dafür sorgen, dass diese nicht ohne Weiteres kommuniziert werden können, ist es für ein niederschwelliges Counseling essenziell, dass Frauen von Frauen und Männer von Männern aus demselben Kulturkreis und wenn möglich aus demselben Herkunftsland beraten werden.

\section{Anonymisierte Fallvignette Deutschland}

Einem jungen Mann, der in einer Flüchtlingsunterkunft alkohol- und drogenabhängig geworden ist, wird nahegelegt, mit einem Counselor zu sprechen. Er berichtet, dass er von seiner Familie nach Deutschland geschickt 
worden sei, als er das Alter erreicht habe, in dem junge Männer in seiner Gegend üblicherweise von den Taliban rekrutiert würden. Die ganze Verwandtschaft habe zusammengelegt, um Schlepper zu bezablen, die ihn nach Europa gebracht hätten. Die Familie habe erwartet, dass er dort rasch Geld verdienen werde und seine Familie unterstützen könne. Weder er noch seine Familie hätten damit gerechnet, dass es Schwierigkeiten geben könne, nachdem er einmal Deutschland erreicht habe. Er habe jedoch nur vier Jahre Schulbildung, Schwierigkeiten die deutsche Sprache zu lernen und sei nicht in der Lage gewesen im Integrationskurs mitzuhalten. Es sei ihm klar geworden, dass er nicht so bald in der Lage sein werde, seine Familie zu unterstützen, und er habe deswegen den Kurs aufgegeben und den Kontakt zu seiner Familie abgebrochen. Der Alkohol und die Drogen hätten ihm anfangs geholfen die Situation zu ertragen.

In diesem Fall sind so viele kulturell vermittelte Schamgefühle involviert, dass es dem Klienten schwerfallen dürfte, einem Therapeuten seine innere Gefühlswelt in Anwesenheit und durch die Vermittlung eines Übersetzers mitzuteilen, so kultursensibel der Therapeut auch vorgehen mag. VBC setzt in einem solchen Fall einen männlichen afghanischen Counselor ein, der eigene Flucht- und Integrationserfahrungen besitzt und sich mit diesen Erfahrungen in der Ausbildung eingehend auseinandergesetzt hat.

\section{Das Beispiel Afghanistan}

Afghanistan ist ein zutiefst religiös geprägtes Land, das in den vergangenen 40 Jahren einer Reihe von gesellschaftlichen Umbrüchen ausgesetzt war, die von Kriegen begleitet wurden. Der kommunistische Staatsstreich von 1978 erschütterte jahrhundertealte Traditionen und brachte kulturelle Einflüsse aus der Sowjetunion in das Land, die bis heute spürbar sind. Der blutige Bürgerkrieg zwischen rivalisierenden Mujaheddin-Gruppen, der dem Abzug der sowjetischen Armee im Jahr 1989 folgte, beförderte den Aufstieg der Taliban, die einen bis dahin ungekannten religiösen Fundamentalismus einführten. Die Rückkehr von Millionen von Flüchtlingen aus Pakistan und dem Iran seit dem Ende der Talibanherrschaft im Jahre 2001 hat das Land ebenfalls kulturell geprägt. Dies gilt jedoch noch stärker für die Internationale Gemeinschaft, deren Gelder an die Umsetzung von Entwicklungszielen gekoppelt werden, die von westlicher Kultur geprägt sind - nicht nur politisch, sondern auch gesellschaftlich.

In Afghanistan wurden Generationen nicht nur von der Gewalt eines 40-jährigen Krieges geprägt, sondern von sehr unterschiedlichen Werten - von Traditionen, die auf einer klaren hierarchischen Familienstruktur und den sozialen Rollen einer kollektivistisch geprägten Gesellschaft mit all ihren Rechten und Pflichten beruhen; von einer Revolution, die gesellschaftliche Zwänge mithilfe kommunistischer Ideale aufbrechen wollte; von dem religiösen Fundamentalismus der Taliban als einer Antwort auf das mörderische Chaos des darauffolgenden
Bürgerkrieges; und seit Beginn diesen Jahrhunderts von gesellschaftlichen Entwicklungen, für die Europa nicht Jahre oder Jahrzehnte benötigte, sondern Jahrhunderte (Missmahl, 2018).

Diese Einflüsse haben nicht nur die Gesellschaft als Ganzes geprägt, sondern Familien Generationskonflikten ausgesetzt, die es notwendig machen, Werte im engsten Kreis neu zu verhandeln. Persönliche, familiäre und gesellschaftliche Werte stimmen oft nicht mehr überein und machen es vielen Menschen schwer, mit sich und anderen in Frieden zu leben. VBC trägt solchen Verhältnissen Rechnung, ist aber kein Allheilmittel für eine zerrissene Gesellschaft. Über die persönliche und familiäre Ebene hinaus ist gesellschaftliches Engagement gefragt, das sich dem Gemeinwohl verschreibt. Das Auswärtige Amt der Bundesrepublik Deutschland fördert daher neben VBC Projekte finanziell, die psychosoziale Kompetenz als Voraussetzung für gesellschaftliche Teilhabe und soziale Integration fördern. In diesem Rahmen werden unter anderem «Sichere Orte» geschaffen, an denen über wichtige, oft sensible Themen offen gesprochen werden kann und an denen eine respektvolle Auseinandersetzung mit traditionellen und modernen Werten stattfindet. Die Arbeit der Projektteams beruht auf dem Menschenbild, welches das VBC leitet, und bewährt sich seit Jahren (Ipso, 2013a, 2014). Der wertebasierte Ansatz hat dafür gesorgt, dass VBC nicht als ideologisch geprägt, sondern als jedem einzelnen Menschen zugewandt wahrgenommen wird.

VBC wurde in den Jahren 2008 bis 2016 als psychosozialer Dienst mit Finanzierunghilfen der Europäischen Union und des Auswärtigen Amtes in das staatliche Gesundheitssystem Afghanistans integriert (Ipso, 2013b, 2013c). Der Beratungsansatz und das darauf aufbauende Curriculum wurden vom afghanischen Gesundheitsministerium übernommen und als verbindliches Qualitätsmerkmal von Psychosozialen Counselor*innen, die im Gesundheitswesen arbeiten dürfen, verankert. VBC wurde in das Basic Package of Health Services (BPHS), das Essential Package of Hospital Services (EPHS) und die National Mental Health Strategy aufgenommen. Im Rahmen des öffentlichen Gesundheitssystems wurden landesweit 380 Stellen für psychosoziale Counselor*innen an Comprehensive Health Centres (CHC) geschaffen sowie Trainings zu psychosozialer Gesundheit für Ärzt*innen, Krankenschwestern und -pfleger durchgeführt. Ärzt*innen können hausintern Patient"innen mit Symptomen an psychosoziale Counselor*innen überweisen, die ursächlich mit einem psychosozialen Stressor in Zusammenhang stehen.

Es handelt sich bei diesen Massnahmen zwar um eine Unterversorgung, da einer Bevölkerung von über 30 Millionen landesweit lediglich ca. 400 Counselor*innen zur Verfügung stehen, jedoch ist das Konzept wegweisend. Um seine Nachhaltigkeit zu gewährleisten, wurden Trainer*innen ausgebildet, die ihrerseits psychosoziale Counselor*innen ausbilden und Weiterbildungen für medizinisches Fachpersonal anbieten können. Zudem wurde ein geeignetes Supervisionssystem entwickelt sowie ein Train-the-Trainer-Programm und Evaluations- und 
Monitoring-Tools, zu denen die Etablierung eines elektronischen Dokumentationssystems gehört.

Hätte das afghanische Gesundheitssystem die nötigen Mittel, könnte es die landesweite psychosoziale Versorgung weiter ausbauen, jedoch ist dies angesichts des chronischen Ressourcenmangels, an dem die staatlichen Dienste Afghanistans leiden, auf absehbare Zeit nicht zu erwarten. Ein vom Auswärtigen Amt finanziertes psychosoziales Zentrum mit einer daran angeschlossenen Tagesklinik in Kabul sorgt dafür, dass 25 weitere Counselor*innen, zwei Psychiater und ein Internist sowie sechs Beschäftigungstherapeut*innen die psychosoziale Versorgung in der Landeshauptstadt verbessern. Pro Woche erhalten zwischen 400 und 500 Jugendliche, Frauen und Männer im Zentrum psychosoziale Unterstützung in Einzelgesprächen und Gruppen. Zudem wird VBC über das Video Online Portal https://www.ipso-ecare.com angeboten, eine ebenfalls vom Auswärtigen Amt finanzierte Massnahme. »Ipso ecare " ermöglicht es ratsuchenden Menschen niederschwellig, ortsunabhängig und diskret über eine sichere Verbindung eine persönliche, psychosoziale Beratung über das Internet zu erhalten.

\section{Ausblick}

Das Beispiel Afghanistan zeigt, dass es möglich ist, die psychosoziale Versorgung in einem von Konflikten, Armut und gesellschaftlichen Umbrüchen geprägten Land nachhaltig zu verbessern, indem mit internationaler Hilfe lokale Ressourcen mobilisiert werden. Zugleich wurden als Antwort auf die hohen Flüchtlingszahlen in Europa in den Jahren 2015 und 2016 in Deutschland 92 Geflüchtete zu psychosozialen Counselor"innen ausgebildet. Sie arbeiten in psychosozialen Beratungszentren in Berlin, Hamburg und Erfurt und sind über das Online Portal https://ipso-care.com erreichbar.

Die Möglichkeiten für den Einsatz von VBC sind jedoch noch vielfältiger. Angesichts der Versorgungsengpässe im deutschen Gesundheitssystem, die zuvor bereits angesprochen wurden, könnte der Beratungsansatz Teil eines mehrstufigen Versorgungssystems bilden, der einer längeren Psychotherapie, die auf einer Diagnostik beruht, vorgeschaltet wäre. Als eine solche vorgeschaltete Intervention könnte VBC einer Chronifizierung psychischer Symptomatik entgegenwirken und dadurch in vielen Fällen eine längere Therapie obsolet machen. Auch in diesem Fall wäre es selbstverständlich wichtig, dass Menschen muttersprachlich und von Counselor*innen aus dem gleichen Kulturkreis beraten werden. Je engmaschiger das Versorgungsnetz wäre, desto feiner könnte das Matching gestaltet werden, das Counselor"innen und Klient*innen die bestmöglichen Voraussetzungen dafür bietet, bereits in der ersten Sitzung die vertrauensvolle, symmetrische Arbeitsbeziehung aufzubauen, die für den Erfolg einer Kurzzeitintervention zentral ist.

\section{Literatur}

Antonovsky, A. (1997). Salutogenese: Zur Entmystifizierung der Gesundheit (dt. erw. Herausgabe von Alexa Franke). Tübingen: dgvt.

Ayoughi, S. (2013). Mental health consequences of war, internal conflict and disrupting living conditions: The case of Afghanistan [Digitale Dissertation]. http://kops.uni-konstanz.de/bitstream/handle/123456 789/24399/Dissertation_S. Ayoughi.pdf? sequence=1\&isAllowed=y: (22.12.2018).

Ayoughi, S., Missmahl, I., Weierstall, R. \& Elbert, T. (2012). Provision of mental health services in resource-poor settings: a randomized trial comparing counselling with routine medical treatment in North Afghanistan (Mazar-e-Sharif). Psychiatry, 12(14). https:// doi.org/10.1186/1471-244X-12-14

Bandura, A. (2007). Self-efficacy: the exercise of control. 9. Aufl. New York: Freeman.

Barwinski, R. (2016). Resilienz in der Psychotherapie: Entwicklungsblockaden bei Trauma, Neurosen und frühen Störungen auflösen. Stuttgart: Klett-Cotta.

Bohus, M. \& Missmahl, I. (2017). Zur Umsetzung alternativer Behandlungsmodelle für Flüchtlinge in der BRD. Was können - was sollten - wir aus Afghanistan lernen? Nervenarzt, 88, 34-39.

Bundesamt für Migration und Flüchtlinge (2018). Aktuelle Zahlen zu Asyl. Ausgabe: September 2018. http://www. bamf.de/SharedDocs/ Anlagen/DE/Downloads/Infothek/Statistik/Asyl/aktuelle-zahlen-zuasyl-september-2018.pdf?_blob=publicationFile (21.12.2018).

Bundesministerium für Familie, Senioren, Frauen und Jugend \& Bundesministerium der Justiz und für Verbraucherschutz (2017). Mehr Schutz bei häuslicher Gewalt. Information zum Gewaltschutzgesetz. https://www. bmfsfj.de/blob/94308/63296d8077e74d1101021f8e9 11e0492/mehr-schutz-bei-haeuslicher-gewalt-data.pdf (22.12.2018).

Bundespsychotherapeutenkammer (2018). Ein Jahr nach der Reform der Psychotherapie-Richtlinie. Wartezeiten 2018. https://www. bptk .de/uploads/media/20180411_BPtK-Studie_Wartezeiten_2018.pdf (22.12.2018).

Curran, C. (1968). Counseling and psychotherapy: the pursuit of values. New York: Sheed and Ward.

Destatis Statistisches Bundesamt. (2018). Bevölkerung und Erwerbstätigkeit. Schutzsuchende. Ergebnisse des Ausländerzentralregisters. https://www. destatis.de/DE/Publikationen/Thematisch/Bevoelkerung /MigrationIntegration/Schutzsuchende2010240167004.pdf?_blob =publicationFile (21.12.2018).

Frankl, V. (2017 [2007]). Ärztliche Seelsorge. Grundlagen der Logotherapie und Existenzanalyse. 7. Aufl. München: dtv.

Gräff-Rudolph, U. \& Sulz, S. (2014). Strategische Kurzzeit-Therapie (SKT) - die erste deutsche Psychotherapie der 3. Welle. Psychotherapie in Psychiatrie, psychotherapeutischer Medizin und klinischer Psychologie, 19(2), 9-26. https://cip-medien.com/wp-content/uploa ds/01.-Graeff-Rudolph.pdf (21.12.2018).

Grawe, K. (2004). Neuropsychotherapie. Göttingen: Hogrefe.

Grawe, K. (2005). (Wie) kann Psychotherapie durch empirische Validierung wirksamer werden? Psychotherapeuthenjournal, 4(1), 4-11.

Heine, S. (2005). Grundlagen der Religionspsychologie. Göttingen: Vandenhoeck \& Ruprecht.

Hijazi, Z. \& Weissbecker, I. (2014). Syria Crisis. Addressing Regional Mental Health Needs and Gaps in the Context of the Syria Crisis. International Medical Corps. https://internationalmedicalcorps.org/ wp-content/uploads/2017/07/Syria-Crisis-Addressing-Mental-Heal th.pdf (21.12.2018).

Hofstede, G. (2001). Culture's consequences: Comparing values, behaviors, institutions, and organizations across nations. 2. Aufl. Thousand Oaks/CA: Sage.

Ipso. (2013a). Peace and stability through cultural dialogue and psychosocial support in Afghanistan. Kabul, Baghlan, Balkh, Herat, Bamiyan, Takhar, Kunduz, Badakhshan, Nangarhar. https://ipso context.org/wp-content/uploads/2015/08/IPSO_CulturalProject_ 19-final.pdf (23.12.2018). 
Ipso. (2013b). Supporting the Integration of Mental Health into the Public Health Care System of Afghanistan by qualifying health professionals and training a pool of national mental health trainers. https:// ipsocontext.org/wp-content/uploads/2015/08/IPSO_MentalHealth _AF004_18.pdf (23.12.2018).

Ipso. (2013c). Mental Health in North Afghanistan. A contribution to peace and reconciliation funded by the German Foreign Office, implemented by Ipso in cooperation with the Ministry of Public Health in Kabul. https://ipsocontext.org/wp-content/uploads/2015/08/IPSO_ Mental_Health_2013_WEB.pdf (23.12.2018).

Ipso. (2014). Afghanistan - Unity in Cultural Diversity. https://ipso context.org/wp-content/docs/AUSSTELLUNGSKATALOG_SCRE EN.pdf (23.12.2018).

Jung, C. G. (1990). Mensch und Kultur. Grundwerk 9. 3. Aufl. Olten: Walter Verlag.

Kirmayer, L. (2007). Psychotherapy and the Cultural Concept of the Person. Transcult Psychiatry, 44(2), 232-257. https://doi.org /10.1177/1363461506070794

Kirschenbaum, H. (2013). Values Clarification in Counseling and Psychotherapy: Practical Strategies for Individual and Group Settings. Oxford: Oxford University Press. https://doi.org/10.1093/ acprof:oso/9780199972180.001.0001

Koch, B. (Hrsg.). (2017). Junge Flüchtlinge auf Heimatsuche. Psychosoziales und pädagogisches Handeln in einem sensiblen Kontext. Heidelberg: Karl-Auer-Verlag.

von Lersner, U. \& Kizilhan, J. (2017). Kultursensitive Psychotherapie (Fortschritte der Psychotherapie 64). Göttingen: Hogrefe Verlag.

Levenson, H. (2017). Brief Dynamic Therapy. 2. Aufl. Washington D. C.: American Psychological Association.

Liedl, A., Böttche, M., Abdallah-Steinkopf, B. \& Knaevelsrud, C. (Hrsg.). (2017). Psychotherapie mit Flüchtlingen - neue Herausforderungen, spezifische Bedürfnisse. Das Praxisbuch für Psychotherapeuten und Ärzte. Stuttgart: Schattauer.

Maslow, A. (1943). A Theory of Human Motivation. Psychological Review, 50, 370-396. https://psychclassics. yorku.ca/Maslow/moti vation.htm (22.12.2018).

Missmahl, I. (2006a). Psychosoziale Hilfe und Traumaarbeit als ein Beitrag zur Friedens- und Versöhnungsarbeit am Beispiel Afghanistans. Psychotheraphie-Forum, 14, 180-185.

Missmahl, I. (2006b). «Window for Life» - Ein Fenster zum Leben. Die psychosoziale Arbeit von Caritas International in Kabul. https:// www.caritas-international.de/beitraege/window-for-life-ein-fensterzum-leben/157289/(21.12.2018).

Missmahl, I. (Juli 2010). Inge Missmabl brings peace to the minds of Afghanistan [TED Talks]. https://www. youtube.com/embed/Jc2F3nawnI (21.12.2018).

Missmahl, I. (März 2016). A human-powered empathy engine [TED Talks]. https://www. youtube.com/watch?v=eqwxR4kNFhk (21.12.2018).

Missmahl, I. (2018). Geflüchtet aus Afghanistan. In W. Machleidt, U. Kluge \& M. Sieberer (Hrsg.), Praxis der interkulturellen Psychiatrie und Psychotherapie. Migration und Psychische Gesundheit (S. 150-153). 2. Aufl. München: Elsevier. https://doi.org/10.1016/ C2016-0-01667-4

Missmahl, I. (i.D.). Psychosoziale Grundversorgung in Afghanistan: ein Beispiel für eine kultursensible Umsetzung von universellen Menschenrechten in einer Gesellschaft im Umbruch. Fortschritte der Neurologie - Psychiatrie. Themenheft Psychiatrie und Menschrechte.

Missmahl, I. \& Ayoughi, S. (2017). Psychosoziale Beratung von Geflüchteten für Geflüchtete - Ein Wissenstransfer von Afghanistan nach Deutschland. In B.T. Koch (Hrsg.), Junge Flüchtlinge auf Heimatsuche. Psychosoziales und pädagogisches Handeln in einem sensiblen Kontext (S. 229-238). Heidelberg: Carl-Auer.

Oerter, R. (2007). Werte - Werthaltungen - Valenzen. In G. Trommsdorff \& H. Kornadt (Hrsg.), Theorien und Methoden der kulturvergleichenden Psychologie (S. 555-615). Göttingen: Hogrefe.

Preitler, B (2016). An ihrer Seite sein. Psychosoziale Betreunng von traumatisierten Flüchtlingen. Insbruck: StudienVerlag.
Quitmann, H. (1996). Humanistische Psychologie: Psychologie, Philosophie, Organisationsentwicklung. 3. überarb. u. erw. Aufl. Göttingen: Hogrefe.

Rezapour, H. \& Zapp, M. (2011). Muslime in der Psychotherapie: ein kultursensibler Ratgeber. Göttingen: Vandenhoeck \& Ruprecht.

Rogers, C. (1994). Die klientenzentrierte Gesprächspsychotherapie. Aus dem Amerikanischen von Erika Nosbüsch. Frankfurt a.M.: Fischer TB.

Stichs, A. (2016). Wie viele Muslime leben in Deutschland? Eine Hochrechnung über die Anzahl der Muslime in Deutschland zum Stand 31. Dezember 2015. Working Paper 71 des Forschungszentrums des Bundesamtes. Nürnberg: Bundesamt für Migration und Flüchtlinge. https://www. bamf.de/SharedDocs/Anlagen/DE/Publi kationen/WorkingPapers/wp71-zahl-muslime-deutschland.pdf? blob=publicationFile (21.12.2018).

Watzlawick, P. (2008 [2001]). Die Konstruktion klinischer «Wirklichkeiten». In P. Watzlawick \& G. Nordone (Hrsg.), Kurzzeittherapie und Wirklichkeit (S. 25-41). München: Piper.

Watzlawick, P. \& Nordone, G. (2008 [2001]). Prolog. In dies. (Hrsg.), Kurzzeittherapie und Wirklichkeit (S. 11-18). München: Piper.

Zaumseil, M. (2006). Einleitung. In E. Wohlfart \& M. Zaumseil (Hrsg.), Transkulturelle Psychiatrie - Interkulturelle Psychotherapie. Interdisziplinäre Theorie und Praxis (S. 4-50). Heidelberg: Springer.

\section{Value-based Counseling. Culture and Religion as a Meaningful Element of a Specific Type of Psychodynamic Short-term Intervention}

Abstract: Value-based Counseling (VBC) evolved from the work experience of the Jungian psychoanalyst Inge Missmahl gained mainly in Afghanistan, but also in Sri Lanka, China, Haiti and Ukraine. The short-term psychodynamic intervention has a salutogenic approach that aims to improve the sense of coherence and self-efficacy of clients in the course of a non-directive but carefully structured conversation. VBC is based on the idea of man/woman as a human being that has an inherent need for leading a meaningful life. Our human potential for self-development and our pursuit of harnessing this potential can be used to facilitate human self-healing. The counseling approach avoids pathologising clinical symptoms underlying intrapsychic or interpersonal conflicts, traumatic experiences, a disruptive social environment, or difficult life transitions such as migration or loss of livelihoods, instead seeking to understand the significance of these symptoms as an expression of unresolved social stress. VBC is based on the experience that we can discover scope for change if we understand our emotional reaction to a situation experienced as an impasse by becoming conscious of the hierarchy of our own values that triggers this emotional reaction as well as of the values of concerned parties. Culture, and in this context religion, shape our perception of the world and of our feelings associated with this perception. Culture and religion are therefore regarded as a resource that can provide meaning and that counselors can refer to and use with deliberate care in their interactions with clients.

Key Words: Afghanistan, collectivism, culture, conflict area, development cooperation, education, easily accessible services, Germany, health system, humanitarian aid, idea of man, meaning, migration, psychosocial counseling, refugees, shortterm psychodynamic intervention, short-term therapy, religion, salutogenesis, self-efficacy, trauma, values 
Value-based counseling (consulenza basata sul valore). Cultura e religione come elemento significativo di un intervento psicodinamico a breve termine

Riassunto: Il Value-based Counseling (VBC) è nato dall'esperienza pratica della psicoanalista junghiana Inge Missmahl, acquisita principalmente in Afghanistan, ma anche in Sri Lanka, Cina, Haiti e Ucraina. L'intervento psicodinamico a breve termine ha un approccio salutogenico volto a ripristinare il senso di coerenza e di auto-efficacia delle persone. Ciò avviene nel quadro di una discussione non direttiva ma attentamente strutturata. Il VBC si basa su un'immagine dell'uomo che parte da un bisogno umano intrinseco di avere una vita piena di senso. Il potenziale di autosviluppo dell'uomo e il suo sforzo di utilizzare questo potenziale costituisce la base per sostenere l'autoguarigione umana. L'approccio di consulenza evita di patologizzare i sintomi clinici basati su conflitti intrapsichici o interpersonali, esperienze traumatiche, un ambiente sociale dirompente o transizioni di vita difficili come la migrazione o la perdita di mezzi di sussistenza, e cerca invece di comprendere il significato di questi sintomi come espressione di stress sociale irrisolto. Il VBC parte dal presupposto che una persona che viene a conoscenza della sua situazione, della gerarchia dei valori ad essa associati e di quelli altrui, nonché dei sentimenti che ne derivano, avrà un margine di manovra per il cambiamento. Come punto di partenza per la percezione del mondo e dei sentimenti ad esso associati, la cultura e, in questo contesto, la religione sono considerate una risorsa significativa che può essere utilizzata specificamente nella consulenza.
Parole chiave: Afghanistan, formazione, consulenza psicosociale, counseling, Germania, cooperazione allo sviluppo, rifugiati, sistema sanitario, aiuto umanitario, collettivismo, cultura, cultura, aree di crisi, migrazione, intervento psicodinamico a breve termine, terapia a breve termine, immagine umana, assistenza a bassa soglia, religione, salutogenesi, autoefficacia, senso, trauma, valori.

\section{Die Autorinnen}

Inge Missmahl ist jungianische Psychoanalytikerin sowie Gründerin und Geschäftsführerin der International Psychosocial Organisation (Ipso) gGmbH.

Birte Brugmann ist promovierte Archäologin, ausgebildete Friedens- und Konfliktberaterin und in der Entwicklungszusammenarbeit, Übergangshilfe und humanitären Hilfe freiberuflich unter anderem für Ipso tätig.

\section{Kontakt}

Inge Missmahl

Ipso gGmbH

Münsterplatz 13

78462 Konstanz

E-Mail: i.missmahl@ipsocontext.org 